\title{
Physical properties of prop-2-en-1-one based single crystals using molecular mechanics
}

\author{
B. N. Anantha Kumar ${ }^{1} \cdot$ M. Ramegowda² $\cdot$ M. B. Nandaprakash ${ }^{3} \cdot$ H. Somashekarappa ${ }^{3} \cdot$ R. Somashekar ${ }^{4}$
}

Received: 31 January 2020 / Accepted: 7 April 2020 / Published online: 20 May 2020

(c) Springer Nature Switzerland AG 2020

\begin{abstract}
A study of mechanical properties of prop-2-en-1-one based compounds using molecular dimension simulations was investigated. Several mechanical properties have been calculated such as elastic constant tensors, shear modulus, bulk modulus, Young's modulus and Poisson's ratio. The s-wave and $p$-wave velocities as well as static dielectric tensor were also calculated. A comparison of these properties with the number of carbon atoms have been carried out to obtain a correlation between molecular structure and mechanical properties and all calculations were performed using GULP program. Correlation between one of the physical parameter and molecular weight has been shown using Functional analysis method. The present study provides a concrete basis to discuss the dominant role played by elastic constants and the critical values associated with these lead to an extent of elastic displacement in the form of 3-dimensional shape which is novel in the context of single crystal analysis. Validation of these results is done in relation to number of carbon atoms and their experimental elastic constants measurements.
\end{abstract}

Keywords GULP · Elastic constant tensor · Prop-2-en-1-one based single crystals · Density of phonon states

\section{Introduction}

Simulation of materials has seen several developments starting from Madelung's constant [1], short range interactions [2], a reasonable estimate of lattice energy [3] and recently the use of atomistic simulation of materials [4]. Several organic single crystal structure data have been reported in various crystallographic journals. Among them we have selected six prop-2-en-1-one based compounds. The mechanical properties of these single crystal calculations were carried out using GULP, a freely available computer programme for symmetry adopted simulation of solids [5]. Using this software crystal properties can be computed [6-11]. The data for the computation have been obtained for the following single crystalline compounds:
1. (2E,2'E)-3,3'-(1,4-Phenylene)bis)1-(4-fluorophenyl)prop-2-en-1-one $\left\{\mathrm{C}_{12} \mathrm{H}_{8} \mathrm{FO}\right\}[6]$.

2. (E)-1-(5-Chlorothiophen-2-yl)-3-(p-tolyl)prop-2-en-1one $\left\{\mathrm{C}_{14} \mathrm{H}_{11} \mathrm{ClOS}\right\}$ [7].

3. (E)-1-(3-Bromophenyl)-3-(3-fluorophenyl)prop-2-en1-one $\left\{\mathrm{C}_{15} \mathrm{H}_{10} \mathrm{BrFO}\right\}$ [8].

4. (E)-3-(3-Methylthiophen-2-yl)-1-p-tolylprop-2-en1-one $\left\{\mathrm{C}_{15} \mathrm{H}_{14} \mathrm{OS}\right\}$ [9].

5. (E)-1-(benzo[d] [1, 3] dioxol-5-yl)-3-(2,3-dichlorophenyl) prop-2-en-1-one $\left\{\mathrm{C}_{16} \mathrm{H}_{10} \mathrm{Cl}_{2} \mathrm{O}_{3}\right\}[10]$.

6. (E)-1-(1,3-Benzodioxol-5-yl)-3-[4-(dimethylamino)phenyl]pprop-2-en-1-one $\left\{\mathrm{C}_{18} \mathrm{H}_{17} \mathrm{NO}_{3}\right\}$ [11].

In the absence of mechanical properties of these single crystals mentioned above we have made an attempt to predict theoretically elastic constants and mechanical

$\triangle$ R. Somashekar, rs@physics.uni-mysore.ac.in | 1 Department of Physics, Government College (Autonomous) Mandya, Mandya 571401, India. ${ }^{2}$ Department of Physics, Government First Grade College, Nanjangud, Karnataka, India. ${ }^{3}$ Department of Physics, Yuvaraja's College (Autonomous), University of Mysore, Mysuru 570005, India. ${ }^{4}$ DOS in Material Science and RIE, University of Mysore, Manasagangotri, Mysuru 570006, India. 
properties. Molecular dynamic simulation GULP program with an input data from ideal crystal structures [6-11] forms the basis for investigating physical parameters dependence on the chemical structure and its variation. In this work, a prediction of elastic constant, shear modulus, bulk modulus, Young's modulus and Poisson's ratio have been carried out. The s-wave and $p$-wave velocities as well as static dielectric constant tensor were also evaluated using molecular dynamics simulation method. We have carried out computational analysis to calculate physical properties of six compounds with the single crystal data reported in IUCr journal. Further the area of concern in this single crystal is stability of the parameters. For example, hydrogen bonded molecular crystalline systems, which are less stable [12]. Here we have calculated the contribution of the zero point energy to the stability of single crystals. There are many theoretical approaches, which we have used empirically parameterized inter atomic potentials to predict morphology and surface related properties [5]. This is computationally a challenging task and reliable force field and crucial in this area. Here, elastic tensor, bulk modulus, dielectric and other physical properties related to second derivatives of the energy with respect to strain and are easily obtained from good single crystal X-ray data. On the basis of Hooke's law the elastic constant are obtained utilizing appropriate crystal symmetry operations. We do comment on the possible realization of the elastic planar displacement of the molecules and their critical nature in the form of 3-dimensional representation of linear, bulk, shear and young's moduli of these crystals.

\section{Materials and methods}

Mechanical properties were simulated using GULP program [12]. There is an added advantage of inclusion of atomic polarisiability through core-shell model [13]. In this study we consider interactions using Backinghan function with appropriate potentials. A simple term to describe electronic polarisiabilty is considered for dielectric properties using core-shell model with appropriate libraries of potentials were used from the catalogue 1992 [14]. Computations were carried out by minimising energy as a function of atomic coordinates and unit cell parameters. Input file parameters were same except for cell parameters, Laue symmetry and fractional coordinates for atoms in the molecule. For generating input files, we have used for species and spring values form the library at www.catlow.lib. In this study we describe cation-O and the $\mathrm{O}-\mathrm{O}$ interactions using a Buckingham function $\mathrm{U}_{\mathrm{ij}}=A \exp \left(\mathrm{r}_{\mathrm{ij}} / \rho\right)-\mathrm{Cij} / \mathrm{r}_{\mathrm{ij}}^{6}+\mathrm{q}_{\mathrm{i}} \mathrm{q}_{\mathrm{j}}$ $e^{2} / r_{i j}$ where the exponential term describes the repulsive energy and the $r^{6}$ term the longer range attraction [15].
For other form of potentials, we refer the reader to the original paper [12].

\section{Results and discussion}

\subsection{Mechanical properties}

We have carried out computational analysis to obtain the physical properties of six compounds with the single crystal data reported in IUCr journal [1-4, 6, 7]. Further the area of concern in this single crystal is stability of the parameters. There are several examples in which hydrogen bonded molecular crystalline systems, are less stable [15]. Here we have calculated the contribution of the zero point energy to the stability of single crystals. There are many theoretical approaches, which we have used empirically parameterized interatomic potentials to predict morphology and surface related properties [5]. This is computationally a challenging task and reliable force field and crucial in this area. Here, elastic tensor, bulk modulus, dielectric and other physical properties related to 2-derivatives of the energy with respect to strain and are easily obtained from good single crystal $X$-ray data. By minimization of the lattice energy we have predicted elastic constant tensor (in $\mathrm{GPa}$ ) and these unique elements are given in the Table 1. In the absence of direct validation of elastic constants, we try to provide conceptual explanation based on experimental elastic constants of equivalent number of carbon atoms compounds. Normally for all organic compounds, the base structure is formed by carbon atoms in this universe and the major contribution to elasticity of compounds do arise from these net work of carbon atoms. Hence we have compared the experimental values of elastic constant with the nearest number of carbon atoms to justify our results predicted here [16-20]. The difference in values is due to the presence of additional ions, causing imperfections in crystals, which do result in the reduction in the values of elastic constants (Fig. 1).

Bulk modulus is maximum for a molecular weight of 323 as predicted from Figs. 2 and 3. Bulk modulus computed using Voigt averaging scheme is given in Table 2 for all the six compounds. Here it is to be mentioned that only compounds 1 and 3 is elastically stable and hence we could compute 3D diagrams for these using ELATE software [http://progs.coudert.name/elate]. These are given in the following figures. These Figs. $2 \mathrm{a}$ and $3 \mathrm{~b}$ indicate that the directional variations of moduli in compounds 1 and 3 . These figures do indicate the gradual changes in the various moduli with respect to displacement to the maximum value corresponding to the lattice constants along $x-, y$ an $d z$-axes. The boundary of these figures indicate the critical values of these moduli and their changes near the 


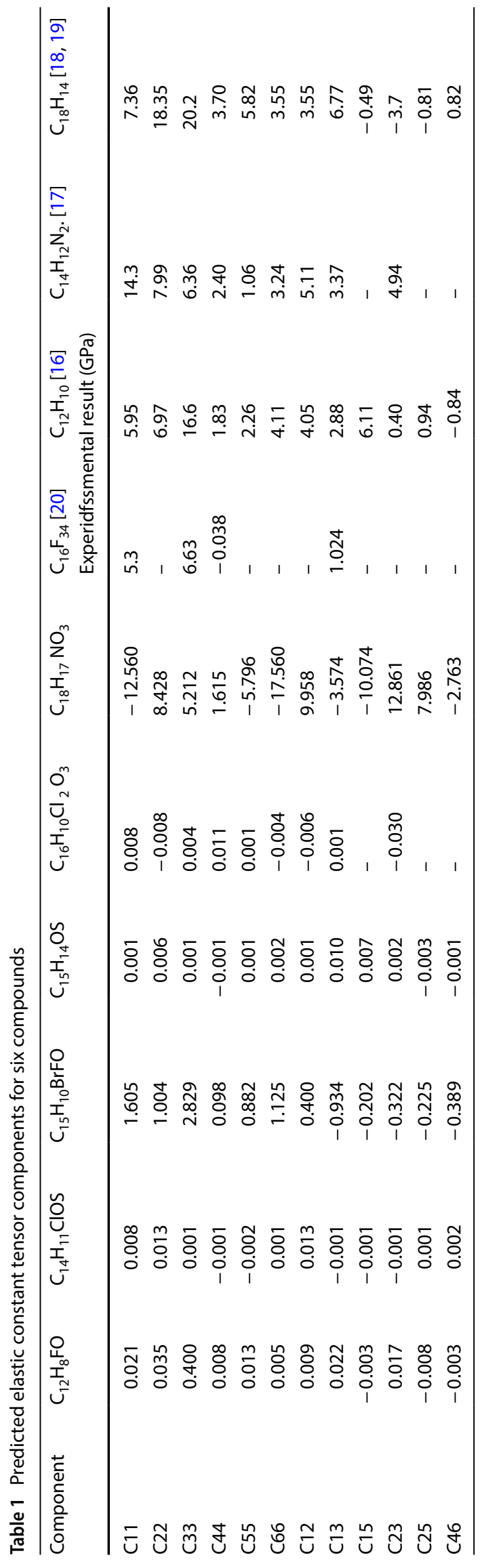

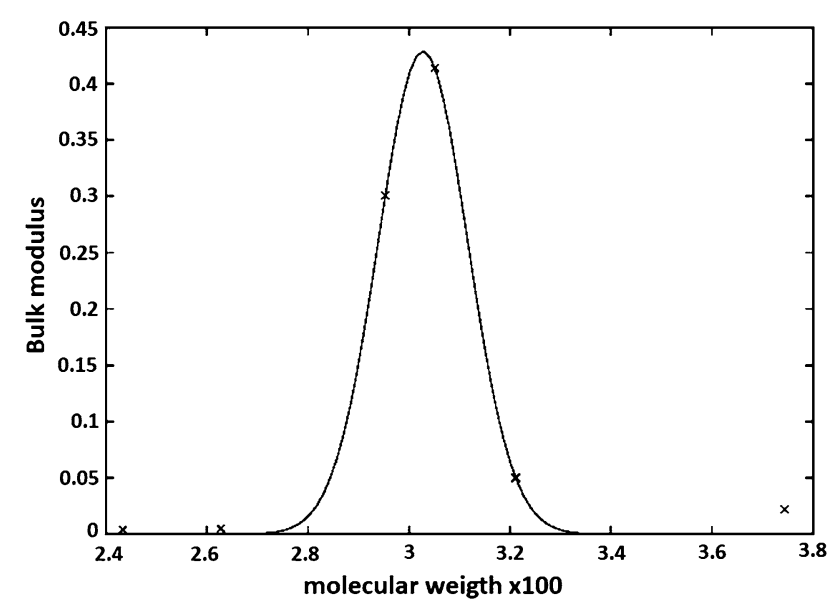

Fig. 1 Variation of bulk modulus with molecular weight of prop-2en-1-one based single crystals

maximum displacement. Normally these values are given by Frank limitations [20].

\subsection{Elastic wave velocities}

Wave velocities are important for the practical applications. These can be determined using the following equations [16-25].

$v_{1}=\sqrt{ }[3 B+4 G / 3 \rho]: v_{t}=\sqrt{ }[G / \rho]$

where $v_{l}$ and $v_{t}$ are longitudinal and transverse velocities. $B$ and $G$ are the bulk and shear moduli, and $\rho$ is the density of material,for the six compounds studied here are given in Table 2 along with $\mathrm{P}$ wave (longitudinal) and S-wave (transverse) velocities. The predicted velocities are higher in compound 3 wherein it has the presence of $\mathrm{Br}, \mathrm{B}$ and Oxygen. In fact for lithium fluoride, the velocity is nearly $2.4 \mathrm{~km} / \mathrm{s}$ which has been reported [26-28] [https://www. rshydro.co.uk/sound-speeds/]. In all these cases, we have relatively lengthy molecules which results in overlap of elastic fields which causes compensation of elastic stress with reversed signs which lead to abrupt in the values and hence observed variation in the elastic wave velocities.

\subsection{Phonon density of states}

By computing vibrational modes and summing over all the states labelled by a single k-point at a particular vibrational energy, we have a diagram which is referred as phonons density of states. Most of the compounds studied here show several distinct peaks except for the sample three. This is due to the elastic stability of the single crystal as evident from the elastic constants (Fig. 4). 


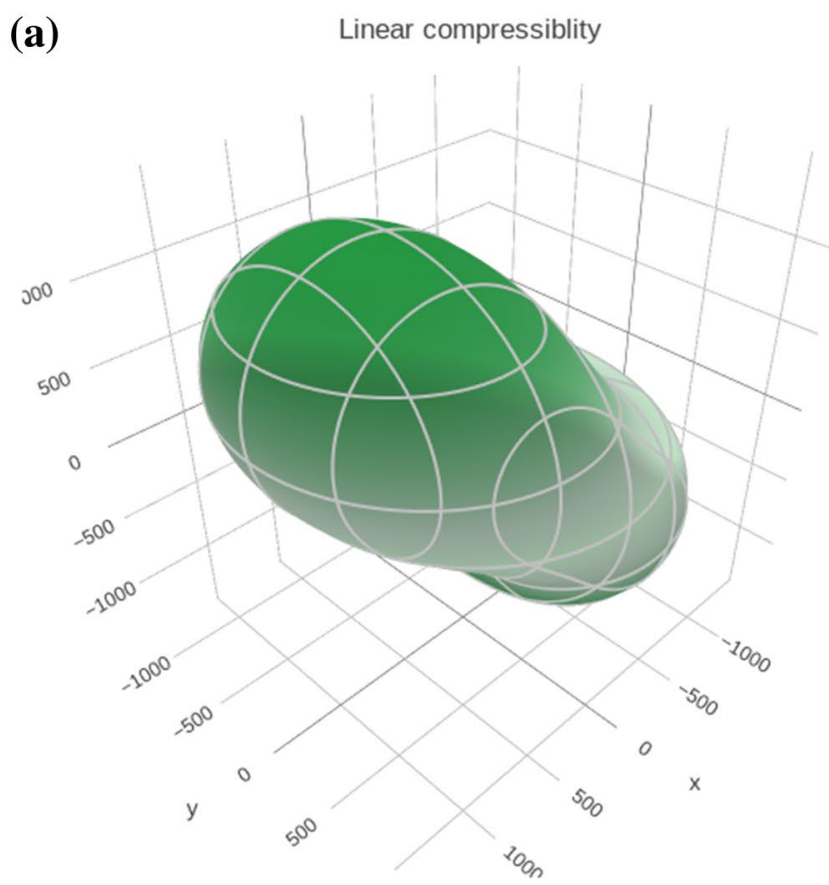

(c)

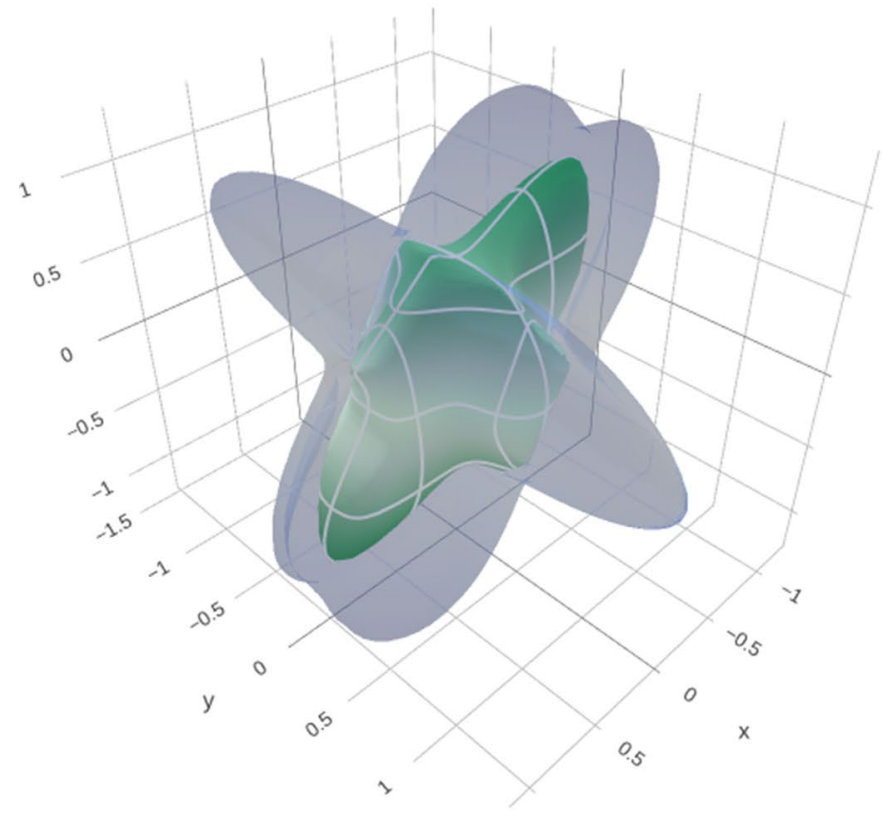

Fig. 2 a Linear b Young c Shear and d Poisson's ratio of Compound 3

\subsection{Electrostatic site potential (ESP)}

ESP is a measure of the Coulomb interaction per unit charge experienced by an ion at a given position in space. ESP is useful quantity in predicting certain (b) Young's modulus

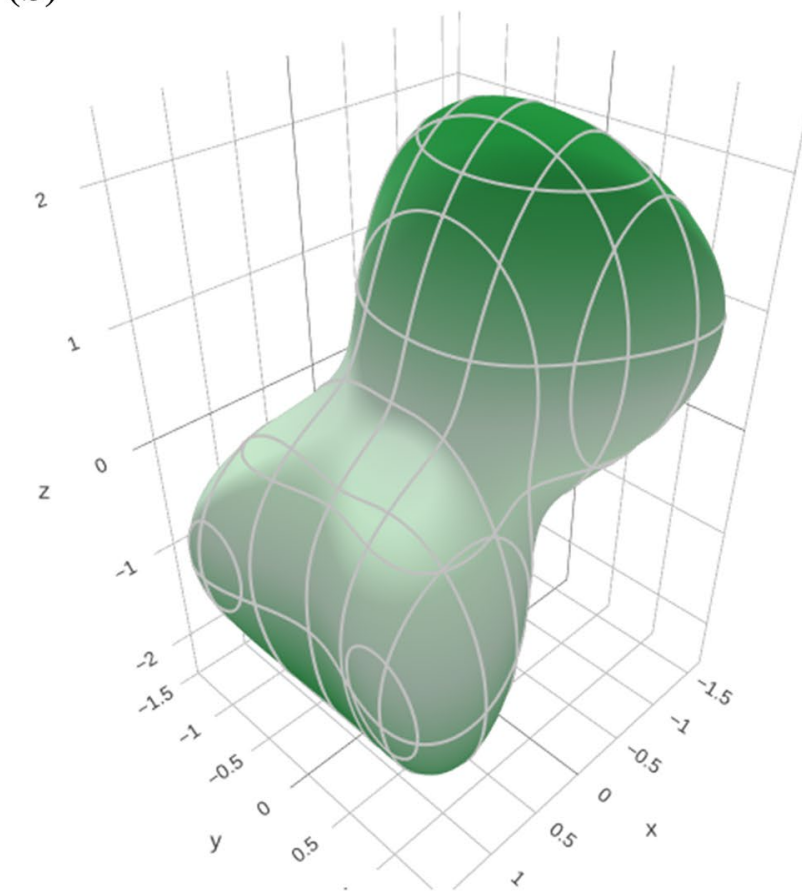

(d)

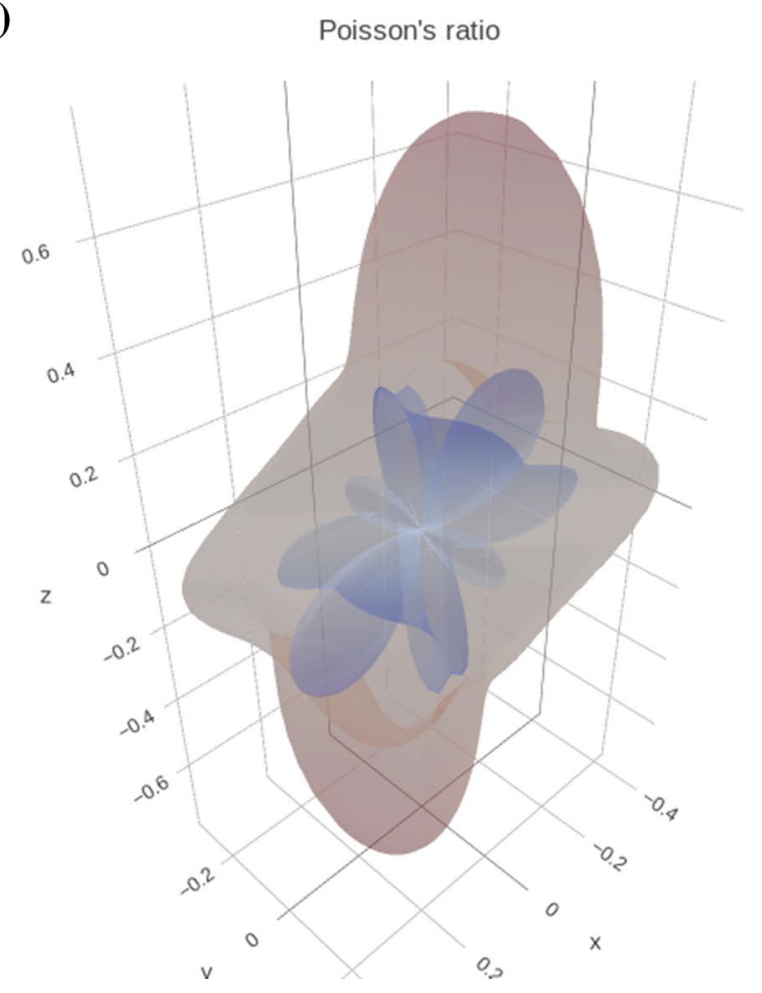

\section{SN Applied Sciences}

properties of a material. Especially for oxygen site, there is a correlation between site potential and basicity. These have been computed from single crystal structure data and are reproduced in the following figure for a better comparison. 
(a)

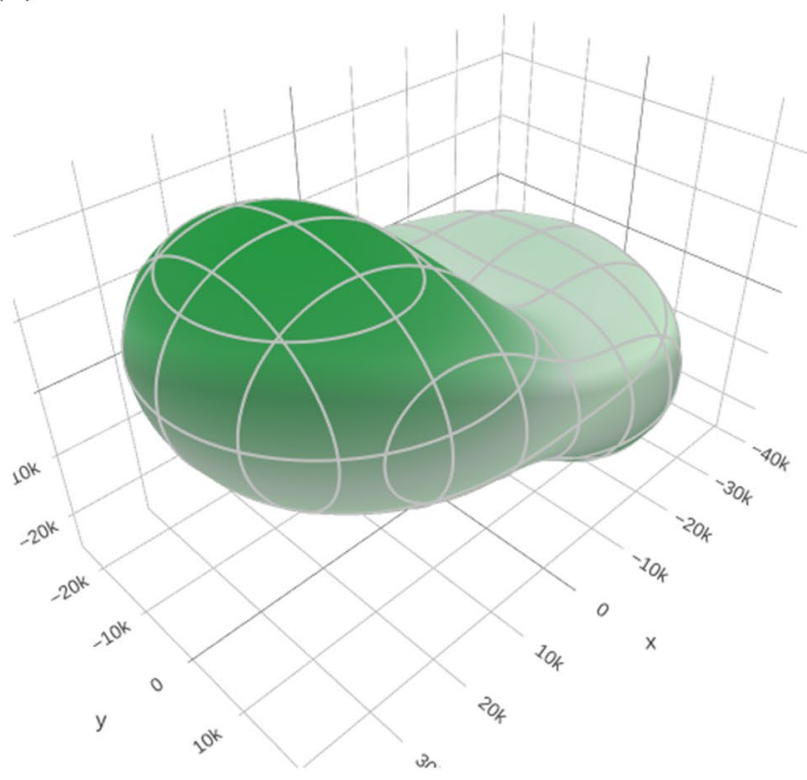

(c)

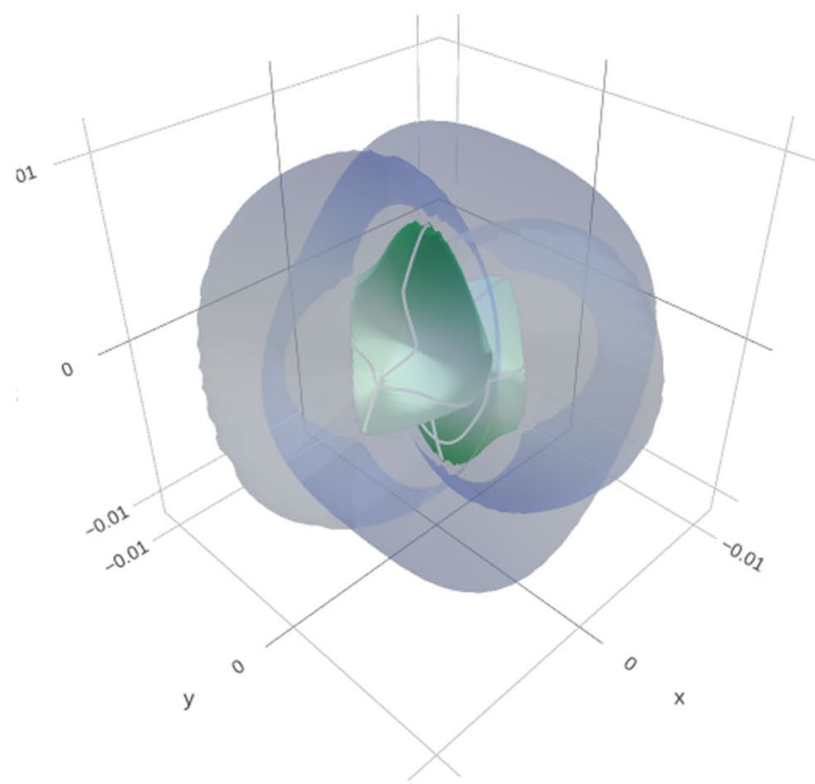

Fig. 3 a Linear b Young c Shear and d Poisson's ratio of Compound 1

For compounds three and six the maximum potential at atomic sites are $0.103 \mathrm{~V}$ and $0.138 \mathrm{~V}$ and a minimum is observed in compound one. Figures 5 and 6 do represent the structural optimisation of the lattice energy.

Predicted electric field gradients at each atomic positions are reproduced in Fig. 6. Here $x$-axis refers to 0:xx, (b)

Young's modulus

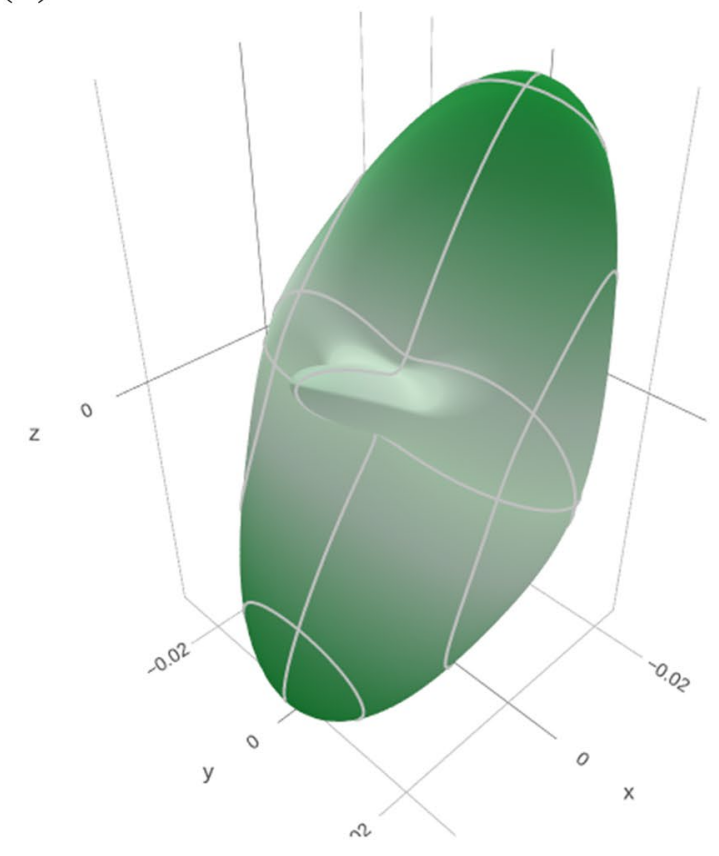

(d)

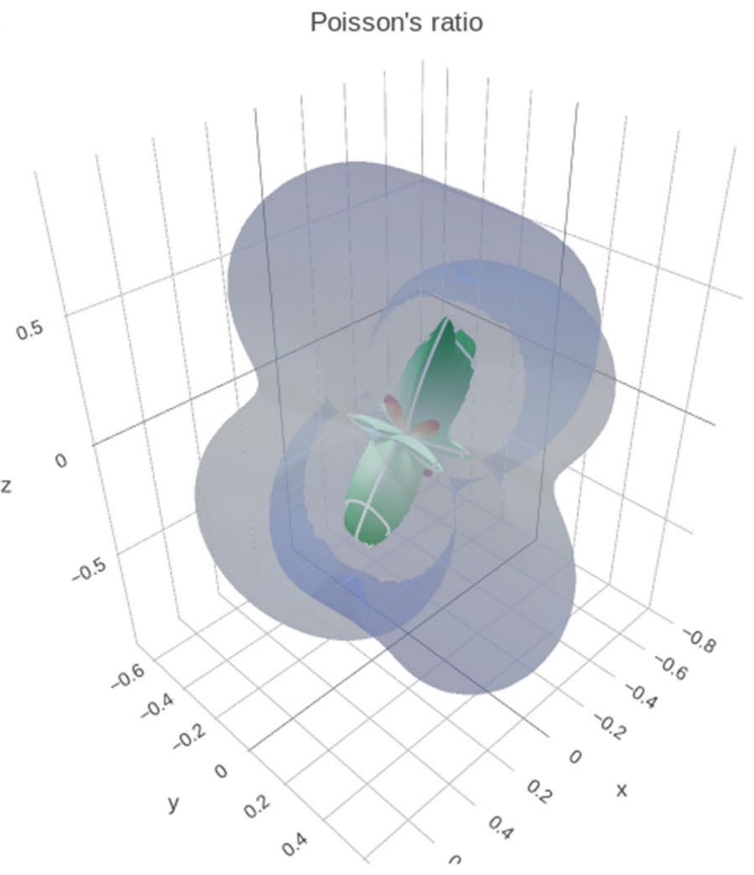

1:xy, 2:yy, 3:xz, 4:yz, 5:zz and y axis refers to atoms. Colour scores is a measure of the electric field gradients (EFG). Important thing to notice in this Fig. 6 , is that the EPG is not same in all the six specimens and presence of heavy elements do alter the nature of interatomic interactions. 
Table 2 Bulk, Young's, Shear moduli and Poisson's ratio for 6-compounds (Voigt averaging scheme)

\begin{tabular}{lllllll}
\hline Parameters & $\begin{array}{l}\mathrm{C}_{12} \mathrm{H}_{8} \mathrm{FO} \\
\mathrm{mw}=374.4\end{array}$ & $\begin{array}{l}\mathrm{C}_{14} \mathrm{H}_{11} \mathrm{ClOS} \\
\mathrm{mw}=262.8\end{array}$ & $\begin{array}{l}\mathrm{C}_{15} \mathrm{H}_{10} \mathrm{BrFO} \\
\mathrm{mw}=305.1\end{array}$ & $\begin{array}{l}\mathrm{C}_{15} \mathrm{H}_{14} \mathrm{OS} \\
\mathrm{mw}=243.3\end{array}$ & $\begin{array}{l}\mathrm{C}_{16} \mathrm{H}_{10} \mathrm{Cl}_{2} \mathrm{O}_{3} \\
\mathrm{mw}=321.14\end{array}$ & $\begin{array}{l}\mathrm{C}_{18} \mathrm{H}_{17} \mathrm{NO}_{3} \\
\mathrm{mw}=295.3\end{array}$ \\
\hline Bulk in GPa & 0.022 & 0.005 & 0.414 & 0.004 & 0.050 & 0.301 \\
Shear in GPa & 0.009 & $7.33 \mathrm{E}-05$ & 1.017 & 0.001 & 0.002 & 0.077 \\
Poisson's & 0.325 & 0.492 & -0.175 & 0.470 & 0.472 & 0.381 \\
Velocity S-wave (km/s) & 0.318 & 0.881 & 12.458 & 0.072 & 0.667 & 0.455 \\
Velocity P-wave (km/s) & 0.714 & 1.057 & 16.479 & 0.301 & 0.179 \\
\hline
\end{tabular}

A plot of Bulk modulus v/s molecular weight can be described by an equation, Bulk modulus $=a^{*} \exp \left(-b^{*}(x-c)^{* *} 2\right) \quad w h e r e, a=0.4288$, $\mathrm{b}=63.75$ and $\mathrm{c}=3.028$

Fig. 4 Phonon density of states (DOS) for prop-2-en-1-one based single crystals

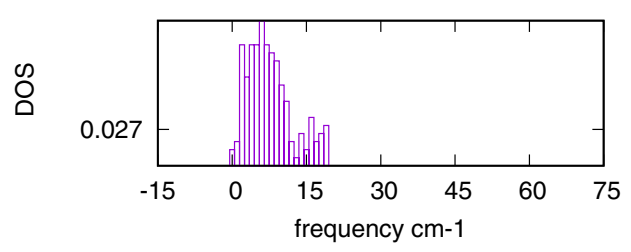

(a)

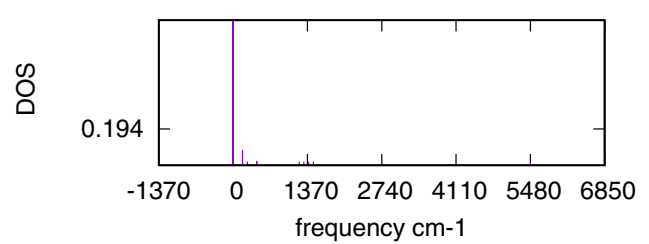

(c)

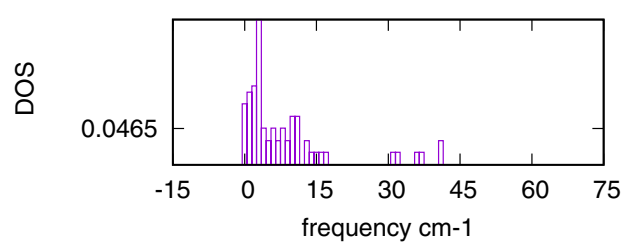

(e)

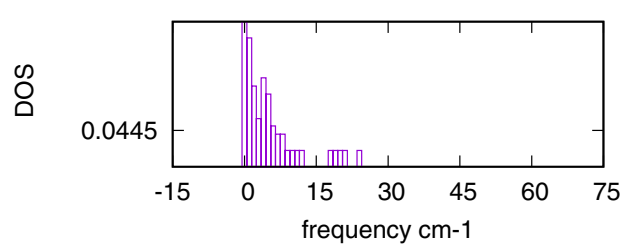

(b)

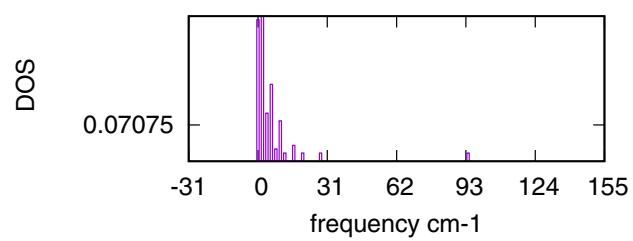

(d)

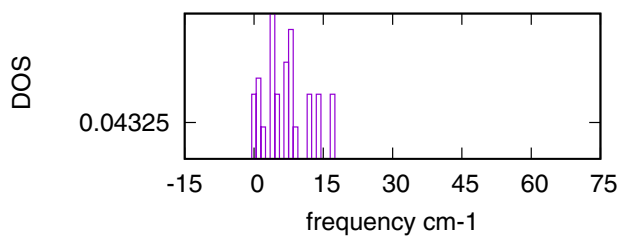

(f)

\subsection{Piezoelectric constant, refractive indices and dielectric constant}

Piezoelectric constant govern correlation between strain and applied electric field for non-centrosymmetric materials which is given by the derivation of induced polarization in a material. These are tabulated in Table 3. Static dielectric constant can be determined by the lattice energy [12] and hence refractive indices by taking the square root of the dielectric constant. As it can be seen from the Table 3 components of dielectric constant and refractive index broadly increases with increase in carbon atoms. In all the cases the piezo electric strain matrix turns out to be zero indicating that these are centrosymmetric molecules.

\subsection{Lattice energy and zero point energy}

In three dimensional crystals, calculation of lattice energy involves interaction of all species within a unit cell and also long and short range potentials. Using GULP we have estimated lattice energy and zero point energy for all the six compounds and are given in Table 3.

\subsection{Functional analysis}

The predicted behavior of $\theta$, which represents a physical parameter, as a function of $t$ for the ith sample

$\hat{\theta}_{i}(\mathrm{t})=\hat{\mu} \mathrm{t}+\sum_{K=1}^{K} \hat{A}_{i k} \hat{\varphi}_{k}(\mathrm{t})$ 


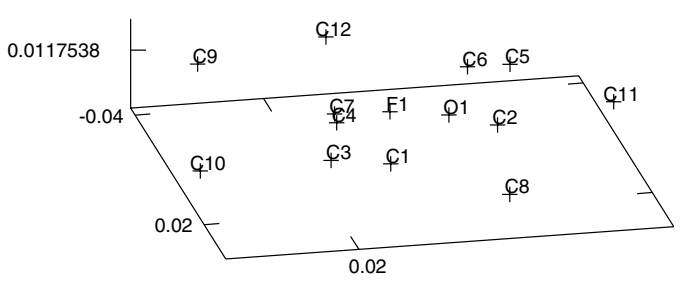

(a)

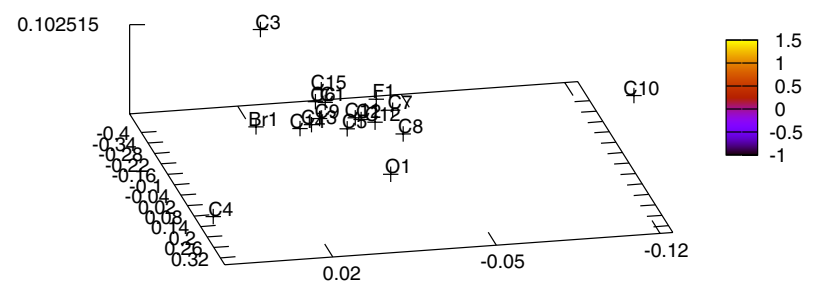

(c)

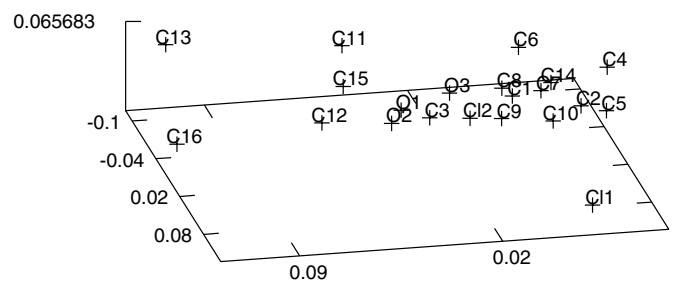

(e)

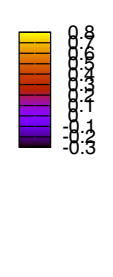

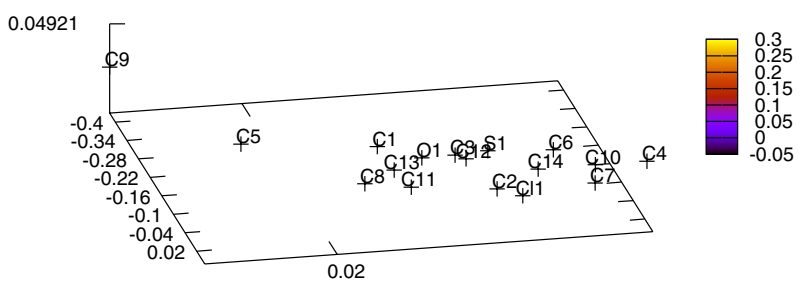

(b)

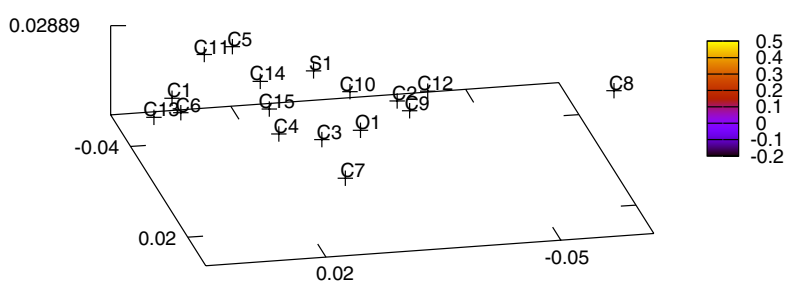

(d)

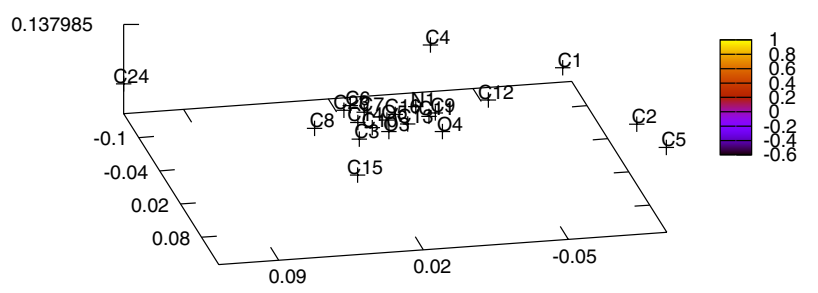

(f)

Fig. 5 Electrostatic potential at each atomic positions for all the six compounds. ( $x-, y$ - and $z$ - axes in derivatives (V/Ang) and fourth axis is potential in (V)

Fig. 6 Electric field gradients (EFG) at each atomic positions in prop-2-en-1-one based single crystals

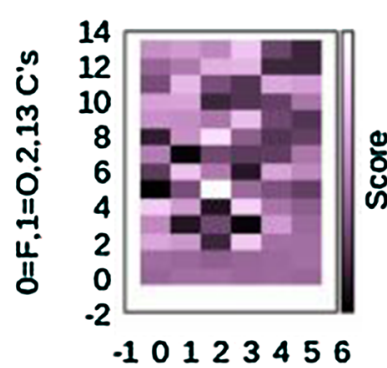

(a)

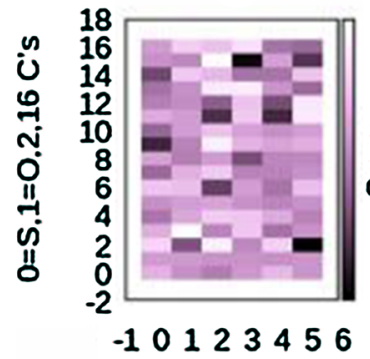

(d)

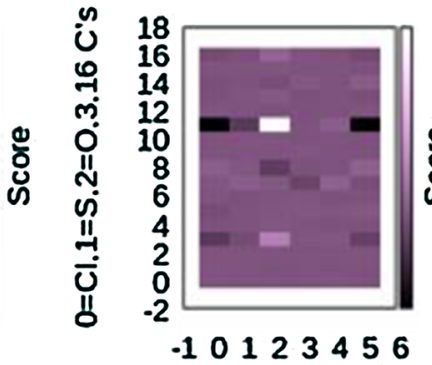

(b)

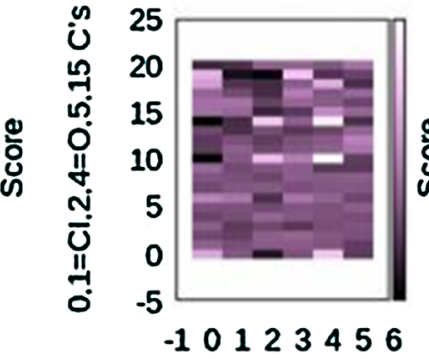

(e)

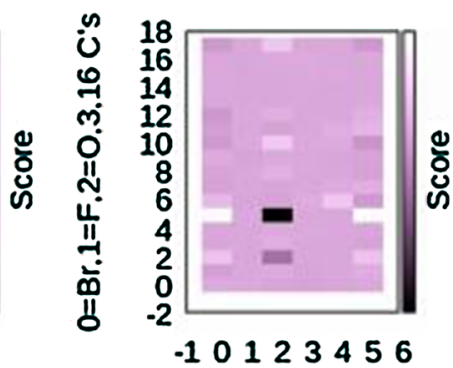

(c)

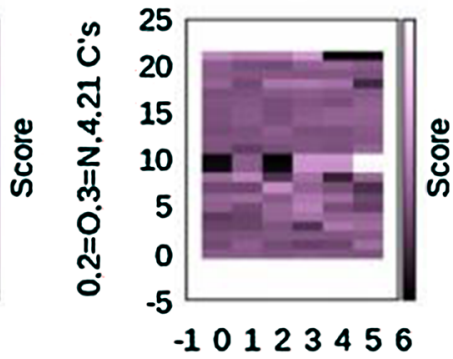

(f) 
Table 3 Predicted parameters using GULP for the size compounds

\begin{tabular}{lcccccc}
\hline Parameter & $\mathrm{C}_{12} \mathrm{H}_{8} \mathrm{FO}$ & $\mathrm{C}_{14} \mathrm{H}_{11} \mathrm{ClOS}$ & $\mathrm{C}_{15} \mathrm{H}_{10} \mathrm{BrFO}$ & $\mathrm{C}_{15} \mathrm{H}_{14} \mathrm{OS}$ & $\mathrm{C}_{16} \mathrm{H}_{10} \mathrm{Cl}_{2} \mathrm{O}_{3}$ & $\mathrm{C}_{18} \mathrm{H}_{17} \mathrm{NO}_{3}$ \\
\hline Lattice energy & $-8.875 \mathrm{eV}$ & $-0.579 \mathrm{eV}$ & $298.17 \mathrm{eV}$ & $-0.781 \mathrm{eV}$ & $-1.732 \mathrm{eV}$ & $131.3 \mathrm{keV}$ \\
Zero point energy & $0.0845 \mathrm{eV}$ & $0.0348 \mathrm{eV}$ & $1.645 \mathrm{eV}$ & $0.0521 \mathrm{eV}$ & $0.0706 \mathrm{eV}$ & $0.0236 \mathrm{eV}$ \\
$\varepsilon$ & 3.382 & 1.518 & 0.621 & 3.059 & 3.243 & 20.638 \\
$\mathrm{n}$ & 1.839 & 1.232 & 0.788 & 1.749 & 1.801 & 4.543 \\
\hline
\end{tabular}

(a)

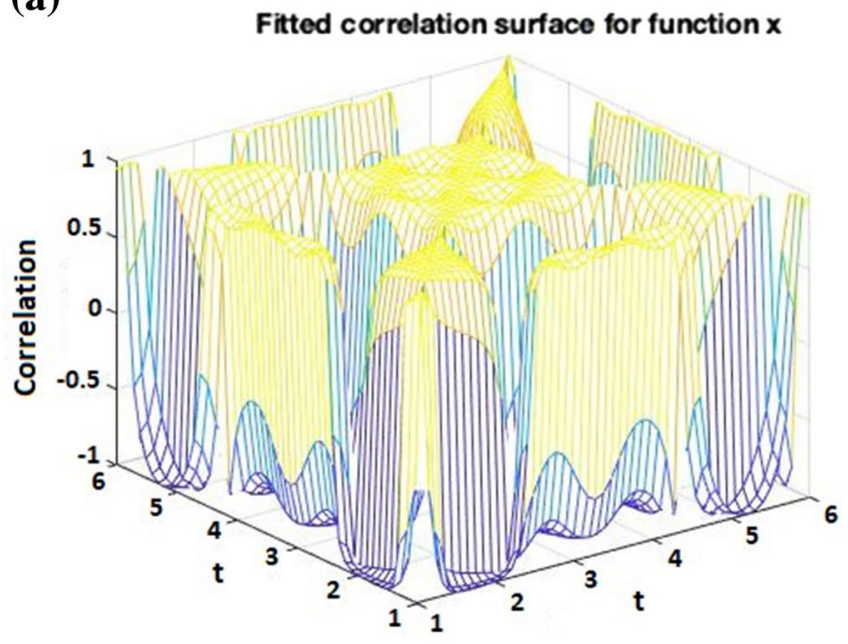

(b)

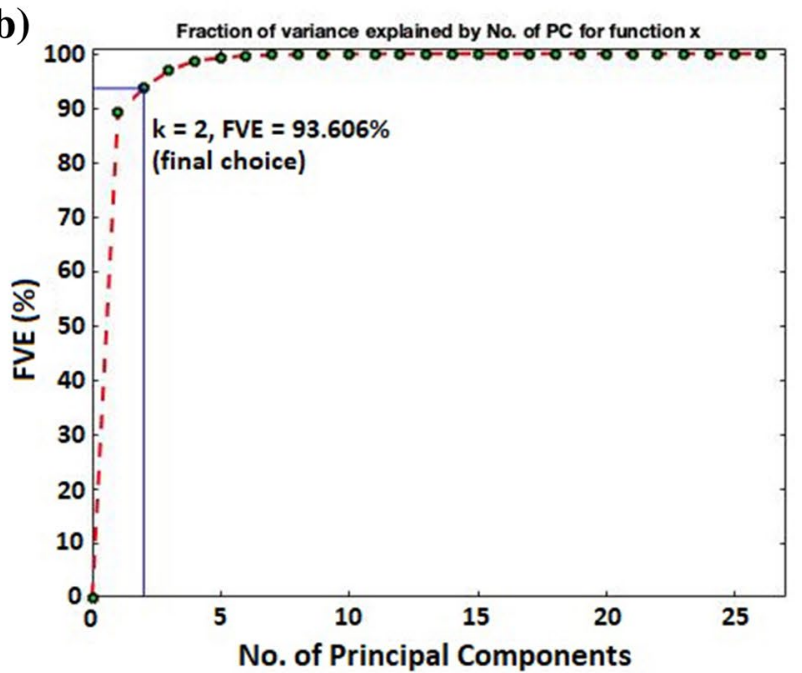

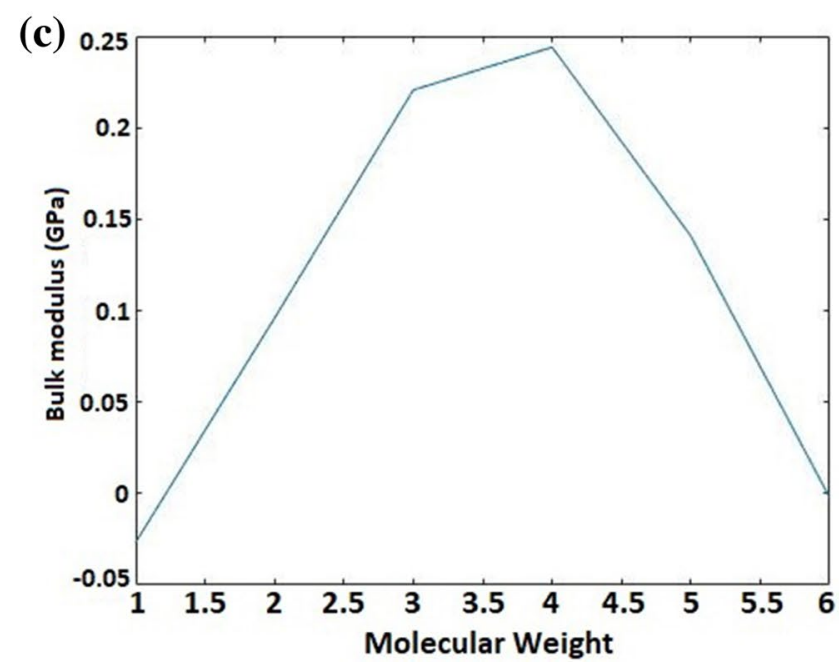

Fig. 7 a Correlation surface with bulk modulus and molecular weight, $\mathbf{b}$ function of variance with molecular weight and $\mathbf{c}$ number of principal components with molecular weight in six samples studied here

where the infinite sum has been truncated to an appropriate finite constant $\mathrm{K}$. The procedures to estimate the FPC, eigenfunctions and eigenvalues are technically involved; for ease of exposition and brevity, the interested reader can refer to Yao et al. [28] for details [23]. Here in Eq. $1 \theta$ refers to measured physical parameter like bulk modulus and " $\mathrm{t}$ " refers to molecular weight of the sampels studied here.
This investigation reports in detail, the results and plots obtained for the hopping length with concentration. Computations were performed using the FPCA package (written in MATLAB:The MathWorks Inc., Natick, MA, USA) available at http://www.stat.ucdavis.edu/PACE/.)

Figure 7 shows the relevance of correlation between the bulk modulus and molecular weight of the six samples studied here. Functional analysis techniques used show 
a correlation over a stretch of the molecular weight of samples and this study also gives a functional mean value of bulk modulus and a maximum observed for the compound three. This procedure also predict values beyond these sample region studied here.

\section{Conclusions}

Using reported Single crystal data for prop-2-en-1-one based compounds we have predicted several physical parameters and the following observations emerge from our study.

1. All physical parameters observed are reasonable from experimental point of view especially with regards to the elastic constants and are in principle agreement with the method used earlier [16-20].

2. Compound 3 and 6 show elastic stability and also directional variation of elastic tensor components.

3. Gaussian distribution of predicted Bulk modulus with molecular weight indicates that the prop-2-en-1-one based compounds have lesser strength of single crystals with higher molecular weight or additional functional groups.

4. S- and P-wave velocities are maximum for compound three and minimum for compound four indicating that the presence of the functional group do play an important role in determining the properties of the material.

5. Phonon density of states computed shows that it is maximum for compound six and minimum for compound three for lower frequencies. This is due to the influence of the functional groups FO and NO respectively.

6. The variation of electrostatic potential values at atomic positions in these six compounds is an indication of the sites of a molecular interactions with the surroundings. The ESP is a tool which is helpful for the study of molecular reactivity in industrial research.

7. Variation in refractive index/dielectric constant, lattice energy and zero point energy are mainly due to molecular weights [22] which do effect the propagation of light.

8. Clear representation of EFG for each atomic positions in prop-2-en-1-one based single crystals which brings out the atomic interactions in different environment.

9. Correlation between one of the predicted physical parameter with molecular weight has been shown using functional analysis technique.

Acknowledgements Authors thank UGC, New Delhi for UPE/CPEPA Projects to the University of Mysore, Mysuru.

\section{Compliance with ethical standards}

Conflict of interest The authors declare that they have no competing interests.

\section{References}

1. Madelung E (1918) Das elektrische feld in systemen von regelmaessig angeordneten punktladungen. Phys Z 19:524-532

2. Born M, Mayer JE (1932) Zur gittertheorie der ionenkristalle. Z Phys 75:1-18

3. Kapustinskii AF (1956) Lattice energy of ionic crystals. Q Rev Chem Soc 10:283-294

4. Gale JD, Rohl AL (2003) The general utility lattice program (LP). Mol Simul 29(5):291-341

5. Gale JD (1997) GULP: a computer program for the symmetry-adapted simulation of solids. J Chem Soc, Faraday Trans 93:629-637

6. Kumar SC, Quah CK, Chandraju S, Lokanath NK, Naveen S, Abdoh M (2017) (2E,2'E)-3,3'-(1,4-Phenyl-ene)bis-[1-(4-fluoro-phen-yl) prop-2-en-1-one]. IUCrData 2:x170238

7. Karthik K, Naveen S, Prabhudeva M, Ajay Kumar K, Lokanath NK, Warad I (2017) (E)-1-(5-Chloro-thio-phen-2-yl)-3-(p-tol-yl)prop2-en-1-one. IUCrData 2:x170038

8. Rajendraprasad S, Kumar C, Quah CK, Chandraju S, Lokanath NK, Naveen S, Warad I (2017) (E)-1-(3-Bromophenyl)-3-(3-fluorophenyl)prop-2-en-1-one. IUCrData 2:x170379

9. Naveen $\mathrm{S}, \mathrm{Al}-\mathrm{Maqtari} \mathrm{H}$, Jamalis J, Sirat HM, Lokanath NK, Abdoh M (2017) (E)-3-(3-Methyl-thio-phen-2-yl)-1-p-tolyl-prop-2-en-1one. IUCrData 2:x170234

10. Naveen S, Dileep Kumar A, Urs D, Ajay Kumar K, Lokanath NK, Warad I (2017) An ortho-rhom-bic polymorph of (E)-1-(benzo[d] [1,3]dioxol-5-yl)-3-(2,3-di-chloro-phen-yl)prop-2-en-1-one. IUCrData 2:x171590

11. Karthik K, Naveen S, Dileep Kumar A, Ajay Kumar K, Lokanath NK, Warad I (2017) An ortho-rhom-bic polymorph of (E)-1-(benzo[d] [1,3]dioxol-5-yl)-3-(2,3-di-chloro-phen-yl)prop-2-en-1-one. IUCrData 2:x162029

12. Mondol NH, Jahren J, Bjorlykke K, Brevik I (2008) Elastic properties of clay minerals. Lead Edge 27:758-770

13. Zheng H, Bailey SW (1994) Refinement of the nacrite structue. Clay Clay Miner 42:46-52

14. Ben Haj Amara A, Ben Brahim J, Plancon A, Ben Rhaiem H (1998) Etude par diffraction $X$ des Modes d'Empilement de la Nacrite Hydratee et Deshydratee. J Appl Cryst 31:654-662

15. Karmous MS (2011) Elastic constant prediction of nacrite: molecular dynamics simulations. Nano Sci Eng 1:62-66

16. Kwiatkowska M, Kowalczyk I, Szymczyk A (2019) Poly(ethylene furanoate) modified with dimerized fatty acid diol towards multiblock copolymers: microstructure-property relationship. Mater Today Commun 20:100577

17. Aranjeira J, Marques $L$ (2020) Role of polyethylene oxide content in polypyrrole linear actuators. Mater Today Commun 23:100906

18. Asadikiya M, Drozd V, Yang S, Zhong Y (2020) Enthalpies and elastic properties of $\mathrm{Ni}$-Co binary system by ab initio calculations and an energy comparison with the CALPHAD approach. Mater Today Commun 23:100905

19. Chena S-B, Yana W-J, Chena Y, Zeng Z-Y, Cheng Y (2020) Enthalpies and elastic properties of $\mathrm{Ni}-\mathrm{Co}$ binary system by ab in functional data analysis for sparse longitudinal data itio calculations and an energy comparison with the CALPHAD approach. Mater Today Commun (in press) 
20. Marx A, Kruger JK, Unruh H (1989) Elastic properties of the premelting phases of the perfluoroalkanes C16F34 and C20F42. Z Phys B 75:101

21. Singh S, Valencia-Jaime I, Pavlic O, Romero AH (2018) Elastic, mechanical, and thermodynamic properties of $\mathrm{Bi}-\mathrm{Sb}$ binaries: effect of spin-orbit coupling. Phys Rev B 97:054108

22. Grima JN, Gatt R, Alaerson A, Evans KE (2005) On the origin of auxetic behaviour in the silicate a-cristobalite. J Mater Chem 15:4003-4005

23. Schroder KP, Sauer J, Leslie M, Catlow CRA, Thomas JM (1992) Bridging hydroxyl groups in zeolitic catalysts: a computer simulation of their structure, vibrational properties and acidity in protonated faujasites ( $\mathrm{H}-\mathrm{Y}$ zeolites). Chem Phys Lett 188:320-325

24. Yoshino M, Takahashi K, Okuda Y, Yoshizawa T, Fukushima N, Naoki M (1999) Contribution of hydrogen bonds to equilibrium a $\beta$ transition of resorcinol. J Phys Chem A 103(15):2775
25. Fleming SD, Rohl AL, Lee MY, Gale JD, Parkinson M (2000) Atomistic modelling of gibbsite: surface structure and morphology. J Cryst Growth 209:159-166

26. Graeme MD, Price SL, Leslie M (2001) Atomistic modelling of gibbsite: surface structure and morphology. Cryst Growth Des 1(1):13-27

27. Wypych G (Ed) (2019) Hand book of solvents, Vol 1, 3rd edn. ISBN 978-1-927885-38-3. Elsevier Publications: Amsterdam

28. Yao E, Muller H, Wang JL (2005) Functional data analysis for sparse longitudinal data. J Am Stat Assoc 100:577

Publisher's Note Springer Nature remains neutral with regard to jurisdictional claims in published maps and institutional affiliations. 